\title{
Resistencia mecánica, tasa respiratoria y producción de etileno de caqui 'Fuyu' durante el almacenamiento' ${ }^{1}$
}

\author{
Saul D ussán-Sarria², Sylvio L. Honório ${ }^{3}$ \& Melissa de L. Matias ${ }^{4}$
}

\begin{abstract}
RESU MEN
El caqui 'Fuyu' presenta escasa revisión de literatura en lo que respecta a propiedades mecánicas y comportamiento fisiológico. En este trabajo fue evaluada la alteración de la resistencia mecánica a la compresión del caqui 'Fuyu' , el comportamiento respiratorio y la evolución de la producción de etileno durante el almacenamiento. Las frutas fueron cosechadas con dos índices de cosecha: Índice 1, frutas cosechadas 15 a 20 días antes de la madurez comercial y, Índice 2, frutas cosechadas con madurez comercial. Las frutas fueron seleccionadas, empacadas en empaque comercial (caja de cartón) y almacenadas bajo dos condiciones de conservación: 1 . Con refrigeración $\left(0 \pm 1{ }^{\circ} \mathrm{C}\right.$ y $65 \pm 1 \%$ de HR; 2 . Sin refrigeración $\left(22 \pm 3^{\circ} \mathrm{C}\right.$ y $71 \pm 6 \%$ de HR). El módulo de elasticidad del caqui disminuye durante el periodo postcosecha independiente de las condiciones de conservación. El módulo de elasticidad varió entre 3.695,3 y 968,2 kPa. El grado de madurez del caqui 'Fuyu' en el momento de la cosecha, influencia los valores del módulo de elasticidad durante el almacenamiento. El caqui 'Fuyu' presenta comportamiento respiratorio característico de fruto climatérico y el pico de evolución de etileno coincide con el pico climatérico.
\end{abstract}

Palabras-clave: frutas, postcosecha, conservación, Diospyros kaki

\section{Mechanical resistance, respiratory rate and the ethylene production of persimmon fruit 'Fuyu' during storage}

\begin{abstract}
The Fuyu persimmon fruit presents little literature review of mechanical properties and physiological behavior. In this paper the alteration of mechanical resistance to compression of persimmon fruit 'Fuyu' the respiratory behavior and the evolution of ethylene production during storage were evaluated. The fruits were harvested with two harvest indexes: Index 1 , fruits with 15 to 20 days before the commercial index, and Index 2, known as the commercial index. The fruits were selected, packed (commercial carton boxes) and stored in two conservation conditions, with refrigeration $\left(0 \pm 1{ }^{\circ} \mathrm{C}\right.$ and $\left.65 \pm 1 \% \mathrm{RH}\right)$ and without refrigeration $\left(22 \pm 3{ }^{\circ} \mathrm{C}\right.$ and $71 \pm 6 \% \mathrm{RH}$ ). The Young's modulus of persimmon fruit decreases during the postharvest period independently of the conservation conditions. The Young's modulus varied between 3,695.3 and 968.2 $\mathrm{kPa}$. The harvest index of persimmon fruit 'Fuyu' influenced the Young's modulus values during the storage. The persimmon fruit 'Fuyu' showed a respiratory pattern characteristic of climacteric fruits and exhibited a maximum ethylene evolution at the climacteric peak.
\end{abstract}

Key words: fruits, postharvest, conservation, Diospyros kaki

\footnotetext{
1 Trabajo hace parte de la tesis de Maestria (Dussán-Sarria, 1998)

2 Departamento de Ingeniería. Universidad Nacional de Colombia. Sede Palmira, Valle del Cauca, Colombia. Carrera 32 vía Candelaria. E-mail: sauldussan@yahoo.com.br

3 FEAGRI/U NICAMP, Cidade Universitária Zeferino Vaz, C.P 6011, CEP 13083-970, Campinas, SP. Fone: (19) 3521 1078. E-mail: honorio@agr.unicamp.br

${ }^{4}$ DTA/UFC. CEP 60511-110, Fortaleza, CE. Fone: (85) 3299 1813. E-mail: mellmatias@gmail.com
} 


\section{INTRODUCCION}

El conocimiento de todas las alteraciones bioquímicas y fisiológicas que suceden durante la maduración del caqui, permiten establecer procedimientos y operaciones de manejo adecuadas, al igual que proyectar equipos y empaques apropiados que eviten o retarden la senescencia del producto, permitiendo así ofrecer en el mercado un producto con la calidad exigida y en el momento oportuno.

La resistencia mecánica de las frutas es una de las principales alteraciones ocurridas durante la maduración y esta relacionada con la textura, que a su vez es condición de firmeza.

De acuerdo con Sams (1999) la textura incluye propiedades mecánicas (dureza, elasticidad y viscosidad), geométricas (tamaño y forma) y químicas (contenido de agua y grasas). En las frutas y hortalizas la textura es función de los organelos celulares y constituyentes bioquímicos, contenido de agua y turgidez de las células y composición de la pared celular. Alteraciones en la textura suceden normalmente durante el crecimiento y desarrollo de los productos hortícolas; implica alteraciones genéticamente programadas en la estructura de la pared celular y otros factores fisiológicos en el desarrollo de la planta.

Suceden alteraciones bioquímicas en la pared celular en los componentes de celulosa, pectinas y hemicelulosa, debido a la acción de enzimas pécticas, especialmente la poligalacturonasa (PG) y pectinametilesterasa (PME). Existen factores ambientales de precosecha que afectan la textura de los productos hortícolas como el clima, los insectos, la fertilización, los patógenos y factores genéticos. En la postcosecha, los principales daños mecánicos en el producto suceden durante el manejo y comercialización originados por impactos, abrasiones y cortes (Sams, 1999; Camargo et al., 2004).

Fuerzas de contacto generalmente ocurren durante la cosecha, el manejo y el almacenamiento de las frutas. Dentro de los ensayos mecánicos evaluados en biomateriales sólidos, el más común y simple es el ensayo de compresión. Sólidos con su forma intacta son sujetos a ensayos de compresión simple axial, empleando el molde cilíndrico rígido o platos planos paralelos (Mohsenin, 1986).

El módulo de elasticidad es uno de los índices utilizados para definir la resistencia mecánica de materiales biológicos y se basa en la teoría de contacto de Hertz, la cual ha sido utilizada para describir la deformación de las frutas (Mohsenin, 1986).

El modulo de elasticidad en caqui (Dussán-Sarria \& Honório, 1998) y la firmeza en melón (Almeida, 2002) han sido utilizados como atributos de evaluación de calidad postcosecha durante el almacenamiento de las frutas. La resistencia mecánica traducida como firmeza es considerada uno de los principales atributos de calidad para exportación de algunas frutas como el melón (Menezes et al., 2000).

La perecibilidad de las frutas está directamente relacionada con la respiración. La respiración es el principal proceso fisiológico que regula la mayoría de las alteraciones bioquímicas que se traducen en alteraciones de color, sabor y aroma. A su vez la tasa respiratoria es función de diversos facto- res intrínsecos y extrínsecos como la variedad de la fruta, el punto de cosecha (grado de madurez), la temperatura ambiente, la concentración de gases alrededor del producto y los daños mecánicos ocasionados en la fruta (Chitarra \& Chitarra, 2005; Ferreira et al., 2005).

El caqui (sin especificar la variedad) es clasificado como fruto climatérico, presentando un aumento considerable de la tasa de dióxido de carbono $\left(\mathrm{CO}_{2}\right)$ y etileno $\left(\mathrm{C}_{2} \mathrm{H}_{4}\right)$ durante la maduración. El punto máximo de liberación de $\mathrm{CO}_{2}$ es conocido como "pico climatérico" cuando las alteraciones mas importantes de la maduración se manifiestan y la gran mayoría de las frutas alcanzan la madurez comercial (Kader, 1992; Watada et al., 1996).

La refrigeración continúa siendo la técnica más económica y eficiente para la conservación prolongada de frutas frescas, siempre asociada a una buena calidad del producto, condiciones adecuadas de conservación, empaque, transporte y comercialización. Durante el almacenamiento se recomienda conservar el caqui a temperaturas entre 0 y $5{ }^{\circ} \mathrm{C}$ y humedad relativa entre 85 y 90\% (Kader, 1992; Sargent et al. 1993).

El caqui variedad Fuyu presenta escasa revisión de literatura en los aspectos aquí abordados, lo que reafirma la necesidad de investigación para este tipo de fruta.

Los objetivos de este trabajo fueron evaluar las alteraciones de la resistencia mecánica a la compresión del caqui ( $D i$ ospyros kaki) variedad Fuyu cosechado con diferentes puntos de cosecha, mantenido a diferentes condiciones de almacenamiento y, evaluar e identificar el comportamiento respiratorio y la evolución de la producción de etileno del caqui durante el periodo de almacenamiento.

\section{MATERIAL Y MÉTODOS}

Fueron utilizados frutos de caqui 'Fuyu', obtenidos de la región de Piedade, Estado de São Paulo, Brasil. Las frutas fueron cosechadas con dos puntos de cosecha (índices de madurez). Índice 1 , frutas cosechadas 15 a 20 días antes de la madurez comercial utilizada normalmente y, Índice 2, frutas cosechadas con madurez comercial.

El caqui con Índice 1 se refiere a una fruta con coloración naranja, firme al tacto y con Índice 2 , con coloración rojiza también firme al tacto. El caqui con madurez comercial (Índice 2) se comercializa en las centrales de abasto de la región. Fueron utilizadas 400 frutas durante todo el experimento.

Las evaluaciones fueron hechas en laboratorios de la Facultad de Ingeniería Agrícola e Ingeniería de Alimentos de la Universidade Estadual de Campinas (UNICAMP), en la ciudad de Campinas, Estado de São Paulo, Brasil.

Las frutas fueron cosechadas con ayuda de una tijera, transportadas a los laboratorios en cajas plásticas, retiradas las impurezas y suciedad con ayuda de un paño seco higienizado, fueron seleccionadas y separadas en lotes de frutas con el mismo punto de cosecha, en seguida fueron empacadas y almacenadas.

Durante el almacenamiento fue utilizado el empaque comercial (caja de cartón) empleado en la comercialización regional. Fueron acondicionadas entre 12 y 15 frutas por empaque y utilizadas 10 frutas/tratamiento en cada ensayo de compresión. 
Fue aplicado un delineamiento enteramente casualizado constituido por cuatro (4) tratamientos, resultantes de frutas con dos puntos de cosecha (Índice 1 y Índice 2) y dos condiciones de conservación (con refrigeración y sin refrigeración). Las condiciones de conservación fueron:

CR - Frutas conservadas con refrigeración, temperatura de $0 \pm 1{ }^{\circ} \mathrm{C}$ y HR de $65 \pm 1 \%$;

SR - Frutas conservadas sin refrigeración, temperatura de $22 \pm 3{ }^{\circ} \mathrm{C}$ y HR de $71 \pm 6 \%$.

Durante el almacenamiento CR y SR fueron utilizados termohigrógrafos y termómetros para registrar la temperatura y humedad relativa (HR). En la refrigeración fue utilizada una cámara fría con control de temperatura. Las frutas mantenidas sin refrigeración permanecieron en el laboratorio en condiciones ambientales.

El módulo de elasticidad fue denominado de aparente por causa de ser un ensayo de compresión de la fruta entera que envuelve diferentes tipos de tejidos con estructuras diferentes como cáscara, pulpa y semillas que denota un modulo de elasticidad global.

En la compresión fue utilizada la prensa universal Ottawa Textura Measuring System (Research Model, Canners Machinery Ltda.). Fue realizada la compresión uniaxial aplicada en la dirección axial de la fruta entera entre platos planos y paralelos con una célula de carga de $50 \mathrm{kgf}$.

La dirección de aplicación de la carga fue definida con base en ensayos preliminares e indica la aplicación de una carga en la misma dirección en la que se encuentran las fibras estructurales del producto.

Los datos fueron adquiridos a través del sistema de adquisición de datos (Aqdados). La velocidad de compresión fue de $17 \mathrm{~mm} \mathrm{~min}^{-1}$. De las diez frutas/tratamiento fueron obtenidos los valores medios de carga y deformación.

Ensayos preliminares también permitieron determinar el módulo de Poisson que resultó ser de 0,4 y el valor de la esfericidad que resultó ser de $88 \%$ permitiendo asumir el caqui como un producto esférico.

Para el cálculo del módulo de elasticidad aparente fue utilizada la eq. (1) definida por Mohsenin (1986) para cuerpos esféricos comprimidos en plato plano.

$$
\mathrm{E}=\frac{0,531 \mathrm{~F}\left(1-v^{2}\right)}{\mathrm{D}^{3 / 2}}\left(\frac{4}{\mathrm{~d}}\right)^{1 / 2}
$$

en que:

E - módulo de elasticidad aparente expreso, $\mathrm{kgf} \mathrm{m}^{-2}$

F - fuerza de compresión aplicada sobre el producto expresa, $\mathrm{kgf}$

$\mathrm{D}$ - deformación en dirección de la fuerza $\mathrm{F}$ expresa, $\mathrm{m}$

$\mathrm{n}$ - coeficiente de Poisson. Adimensional igual a 0,4

$\mathrm{d}$ - diámetro equivalente del caqui expreso en $\mathrm{m}$, siendo $d=\left(d_{1} d_{2} d_{3}\right)^{1 / 3}$

$\mathrm{d}_{1}, \mathrm{~d}_{2}, \mathrm{y} \mathrm{d}_{3}$ son tres diámetros mutuamente perpendiculares de la fruta expresos en $m$

Para convertir kgf m ${ }^{-2}$ en $\mathrm{kPa}$ se multiplica por 0,0098000196. Los valores de $\mathrm{E}$ en este trabajo son presentados en $\mathrm{kPa}$. Los valores de la fuerza $\mathrm{F}$ fueron obtenidos en los puntos medios de las curvas de fuerzas vs. deformación.
Además de analizar y discutir el comportamiento del módulo de elasticidad del caqui durante el almacenamiento, también fue hecha análisis de variancia a través del teste $\mathrm{F}(1 \%)$ y comparación entre medias a través de la prueba de Tuckey (1\%) entre los tratamientos, considerando los valores medios del módulo de elasticidad del tratamiento considerado crítico en la conservación del producto.

El análisis de gases fue hecho en un cromatógrafo de gases Varian 3400, con detector de conductividad térmica para medir $\mathrm{CO}_{2}$ y $\mathrm{O}_{2}$ y un detector de ionización por llama para medir la producción de $\mathrm{C}_{2} \mathrm{H}_{4}$.

El cromatógrafo fue equipado con dos columnas: A) una columna de $10 \mathrm{~cm}$ de silica gel para remover el agua a través de una columna de $183 \mathrm{~cm} \times 0,3 \mathrm{~cm}$ que contiene Chromosorb 106 (60 a 80 mesh) para separar $\mathrm{CO}_{2}$ y $_{2} \mathrm{H}_{4}$; B) una columna de $366 \mathrm{~cm} \times 0,3 \mathrm{~cm}$, conteniendo una malla molecular 5A (45 a 60 mesh) para separar $\mathrm{O}_{2}$ y $\mathrm{N}_{2}$, mas $274 \mathrm{~cm}$ de tubo vacío reteniendo el $\mathrm{O}_{2} \mathrm{y} \mathrm{N}_{2}$ hasta que el etileno sea cuantificado.

Ambas columnas estaban en paralelo. En la columna A fue colocado $1 \mathrm{~mL}$ de muestra de gas; al mismo tiempo en la columna $\mathrm{B}$, fueron colocados $100 \mathrm{~mL}$ de muestra. Las temperaturas del inyector, columna, detector de ionización por llama y el detector de conductividad térmica, fueron respectivamente $60,80,200$ y $120^{\circ} \mathrm{C}$.

Las tasas de flujo del helio, hidrógeno y aire fueron de 30 , 29 y $300 \mathrm{~mL} \mathrm{~min}^{-1}$ respectivamente. Las tasas de producción de $\mathrm{CO}_{2}$ y $\mathrm{C}_{2} \mathrm{H}_{4}$ fueron calculadas y graficadas en función del tiempo.

Se optó por monitorear la producción de gases del caqui con punto de cosecha comercial mantenido a $22 \pm 3{ }^{\circ} \mathrm{C}$ y HR de $71 \pm 6 \%$, por representar la condición experimental más drástica y realista. Los resultados de la producción de $\mathrm{CO}_{2} \mathrm{y}$ $\mathrm{C}_{2} \mathrm{H}_{4}$ fueron expresados como la media de tres repeticiones, cada repetición con masa media de $1 \mathrm{~kg}$ de frutas siendo colectadas muestras a cada $48 \mathrm{~h}$.

\section{RESULTADOS Y DISCUSIÓN}

Los valores medios del módulo de elasticidad de caqui durante el período de almacenamiento son observados en la Figura 1. Las frutas fueron evaluadas hasta el período límite de conservación en el cual el caqui aún presentaba condiciones de evaluación. Considerando todos los tratamientos en general, el módulo de elasticidad varió entre 3.695,3 a 968,2 $\mathrm{kPa}$. Estos valores de firmeza fueron influenciados por el índice de madurez de las frutas (Ferreira et al., 2005; Camargo et al., 2004).

Obsérvese que el caqui con madurez comercial mantenido sin refrigeración (I2SR) presentó el período de conservación más corto comparado con las demás condiciones evaluadas, siendo ésta la condición crítica de conservación que se traduce en menor vida útil del producto.

Considerando la condición crítica que corresponde a 17 días de almacenamiento, fue fijado este período y realizado el análisis de variancia y comparación entre medias entre los tratamientos. 


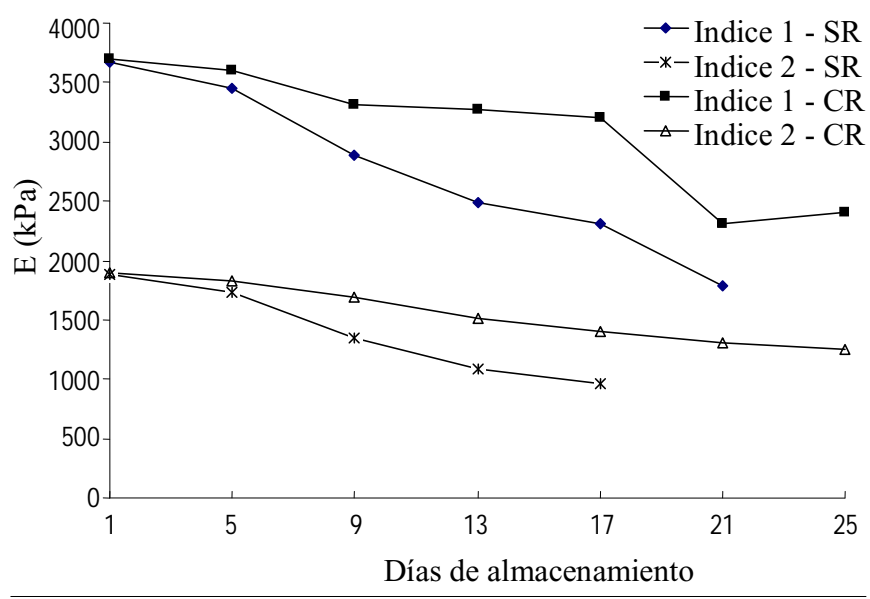

Figura 1. Valores del módulo de elasticidad aparente del caqui 'Fuyu' durante el almacenamiento bajo diferentes puntos de cosecha y condiciones de conservación

Los valores medios del módulo de elasticidad del caqui cosechado con el mismo punto de cosecha conservado con y sin refrigeración no presentaron diferencia estadística a nivel de significancia del $1 \%$, o sea, en el período evaluado, no se observó el efecto de la refrigeración en el mantenimiento de la firmeza de la fruta.

Los valores del módulo de elasticidad de caqui con diferente punto de cosecha, almacenados con las mismas condiciones de refrigeración presentaron diferencia estadística a nivel de significancia del 1\%, lo que significa que existió efecto significativo del grado de madurez de las frutas en el mantenimiento de la firmeza del producto durante el almacenamiento. El caqui con Índice 1 , cosechado 15 a 20 días antes de la madurez comercial, además de continuar con el proceso de maduración durante el almacenamiento, presenta mayor resistencia mecánica a la compresión que el caqui cosechado con madurez comercial.

Verificase que la producción de $\mathrm{CO}_{2}$ durante los primeros 5 días posteriores a la cosecha presentó pequeña variación, valores entre 10,2 y $12,8 \mathrm{mg} \mathrm{CO}_{2} \mathrm{~kg}^{-1} \mathrm{~h}^{-1}$ (Figura 2). La elevación de la tasa respiratoria ocurrió a partir del $6^{\circ}$ día, alcanzando el pico climatérico en el $13^{\circ}$ día posterior a la cosecha. Posteriormente, se observo un

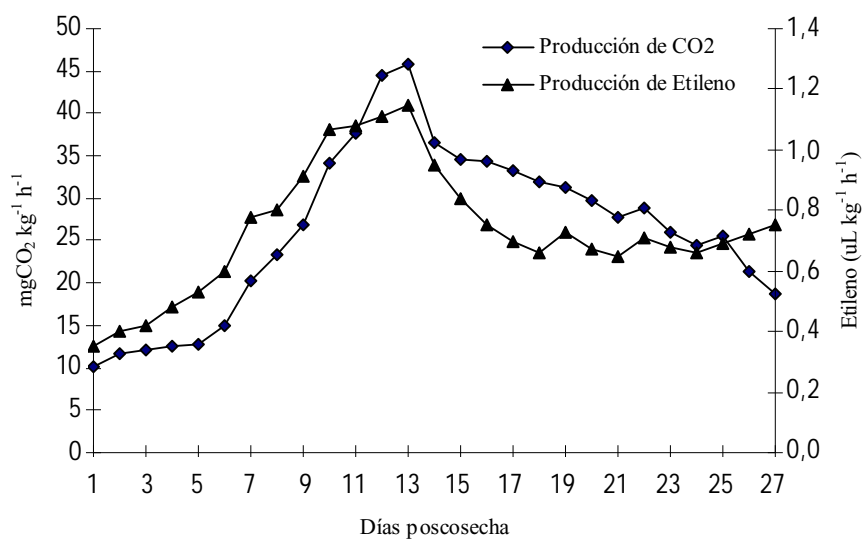

Figura 2. Producción de $\mathrm{CO}_{2}$ y etileno de caqui 'Fuyu', Índice 2, almacenado a $22 \pm 3{ }^{\circ} \mathrm{C}$ y HR de $71 \pm 6 \%$ período decreciente hasta el final del experimento, y con valores nunca inferiores a $18 \mathrm{mg} \mathrm{CO}_{2} \mathrm{~kg}^{-1} \mathrm{~h}^{-1}$.

El pico de producción de $\mathrm{C}_{2} \mathrm{H}_{4}$ (Figura 2) coincidió con el pico climatérico, decreciendo posteriormente hasta el $18^{\circ}$ día después de la cosecha, y se mantuvo con pequeña tasa de variación hasta el $27^{\circ}$ día.

La tasa de respiración varió aproximadamente de 10 a $46 \mathrm{mg}$ $\mathrm{CO}_{2} \mathrm{~kg}^{-1} \mathrm{~h}^{-1}$ a una temperatura de $22 \pm 3{ }^{\circ} \mathrm{C}$. Valores aceptables en comparación con los encontrados por Kader (1992) para caqui 'Fuyu' y por Itamura et al. (1991) para caqui 'Hiratanenashi' que fueron de 20 a $24 \mathrm{mg} \mathrm{CO}_{2} \mathrm{~kg}^{-1} \mathrm{~h}^{-1}$ a una temperatura de $20^{\circ} \mathrm{C}$.

Las diferencias seguramente son debidas al efecto de la temperatura. Situación similar sucedió con las tasas de $\mathrm{C}_{2} \mathrm{H}_{4}$, las cuales variaron de 0,35 a $1,15 \mathrm{~mL} \mathrm{~kg}^{-1} \mathrm{~h}^{-1}$ a $23 \pm 1{ }^{\circ} \mathrm{C}$ y segundo Kader (1992), a $20^{\circ} \mathrm{C}$ para caqui 'Fuyu' variaron de 0,1 a $0,5 \mathrm{~mL}$ $\mathrm{kg}^{-1} \mathrm{~h}^{-1}$. Estos resultados muestran que el caqui presenta durante la maduración un comportamiento respiratorio característico de fruto del grupo climatérico. En general, el pico climatérico está asociado a mejor calidad de las frutas de esa categoría. Según Dussán-Sarria (1998), fue verificada una correspondencia entre el pico climatérico presentado en este trabajo y el periodo adecuado para consumo en fresco de esta fruta.

Analizándose el comportamiento respiratorio en relación al módulo de elasticidad, se observó que el decrecimiento en la resistencia mecánica del caqui 'Fuyu' (Figura 1) se acentuó a partir del $5^{\circ}$ día después de la cosecha, prácticamente al mismo tiempo que comenzó la ascensión climatérica (Figura 2). Paralelamente hubo aumento de la tasa de producción de $\mathrm{C}_{2} \mathrm{H}_{4}$, que entre el $6^{\circ}$ y $7^{\circ}$ día ya había prácticamente doblado la tasa de producción, ciertamente ejerciendo efecto sobre la firmeza del caqui. El etileno es considerado un activador del mecanismo de ablandamiento de frutos de caqui (Itamura et al., 1991).

\section{CONCLUSIONES}

1. Independiente del punto de cosecha y de las condiciones de conservación de los frutos de caqui 'Fuyu', el módulo de elasticidad aparente disminuyó considerablemente en el período postcosecha.

2. El módulo de elasticidad durante la postcosecha de caqui 'Fuyu' es influenciado por el grado de madurez en el momento de la cosecha.

3. El caqui 'Fuyu' presenta comportamiento respiratorio característico de fruto climatérico.

4. El pico de evolución de etileno coincide con el pico climatérico.

\section{LITERATURA CITADA}

Almeida, A. S. Conservação de melão cantaloupe 'Hy-Mark' tratado com 1-MCP após a colheita. Mossoró: ESAM, 2002. 143p. Dissertação Mestrado

Camargo, G. G. R; Ferreira, M. D; Andreucetti C. Testes de impacto em laboratório para tomate de mesa (Lycopersicon esculentum mill.), cultivar Débora. Horticultura Brasileira, v.22, n.2, suplemento CD-Rom 2004. 
Chitarra, M. I. F.; Chitarra, A.B. Pós-Colheita de frutas e hortaliças: Fisiologia e manuseio. 2.ed. Lavras: UFLA, 2005. 783p.

Dussán-Sarria, S. Comportamento pós-colheita de caqui ( $\mathrm{Di}$ ospyros kaki): Avaliação física e química. Campinas: UNICAMP, 1998. 72p. Dissertação Mestrado

Ferreira, M. D., Franco, A. T. O., Kasper, R. F. Qualidade do tomate de mesa em função da época de colheita. Scientia Agricola, v.62, n.5, p.446-451, 2005.

Itamura, H.; Kitamura, T.; Taira, S.; Harada, H.; Ito, N.; Takahashi, Y.; Fukushima, T. Relationship between fruit softening, ethylene production and respiration in Japanese persimmon 'Hiratanenashi'. Journal of Japanese Society for Horticultural Science, v.60, n.3, p.695-701, 1991.

Kader, A. A. Postharvest technology of horticultural crops. 2.ed. California: University of California, 1992. 296p.
Menezes, J. B.; Filgueiras, H. A. C.; Alves, R. E.; Maia, C. E.; Andrade, G. G.; Almeida, J. H. S.; Viana, F. M. P. Características do melão para exportação. Melão pós-colheita. Frutas do Brasil (10), Brasilia: Embrapa SPI. 2000. p.13-22.

Mohsenin, N. N. Physical properties of plant and animal materials: Structure, physical characteristics and mechanical properties. 2.ed. New York: Gordon and Breach Science Publishers Inc., 1986. p.225-382.

Sams, C. E. Preharvest factors affecting postharvest texture. Postharvest Biology and Technology, v.15, p.233-247, 1999.

Sargent, S. A.; Crocker, T. E.; Zoellner, J. Storage characteristics 'Fuyu' persimmons. Proceedings of the Florida State Society for Horticultural Science, v.106, p.131-134, 1993.

Watada, A. E.; Ko, N. P.; Minott, D. A. Factors affecting quality of fresh cut horticultural products. Postharvest Biology and Technology, v. 9, p. 115-125, 1996. 